\title{
THE CURRENT ROLE OF COBALT-60 TELETHERAPY IN CANCER CARE
}

\author{
V Ramanathan ${ }^{1}$ \\ Department of Radiography \& Radiotherapy, Faculty of Allied Health Sciences, General Sir John Kotelawala \\ Defence University, Ratmalana, Sri Lanka ${ }^{1}$
}

\begin{abstract}
Cobalt-60 teletherapy for cancer treatment is becoming less common. It is due to advances in Linac technology, such as beam collimation, conformal radiation therapy, and intensity modulation. Despite this, most developing countries rely on Cobalt teletherapy because of low prices, scarcity of skilled medical professionals, and uncomplicated treatment procedures. The goal of this study was to see how Cobalt teletherapy is used in cancer care around the world right now-the IAEA-DIRAC database to gather information for this investigation. Nearly every country has some radiation facility in the DIRAC areas. Other data gathered for all countries in DIRAC regions included the number of teletherapy machines and each country's population and income group. For every DIRAC region (high income, upper middle income, lower middle income, and low income), this chart information shows how many megavoltage treatment units are currently available. Within each income bracket, linear accelerators and Cobalt teletherapy machines were distributed in 2006, 2013, and 2020, respectively. Around the globe, there are currently 1766 Cobalt teletherapy units available, or 12.7\% of all available external beam radiotherapy machines. The majority of Cobalt teletherapy units located in developing countries are about to 86 percent. Megavoltage machine per million people varies widely around the world. Currently, there is a 0.12 to 9.11 megavoltage machine per million population range available, which is supplemented by 0.04 to 1.41 from Cobalt teletherapy. Even though there is a declining trend, the use of Cobalt teletherapy is unavoidable in low-and middleincome countries to manage the increasing number of new cancer cases.
\end{abstract}

KEYWORDS: Radiotherapy, Cobalt-60 teletherapy, IAEA-DIRAC database, DIRAC region, Megavoltage units per million population, Linear accelerators

Corresponding Author: V Ramanathan, Email: vijitha.r@kdu.ac.lk 


\section{INTRODUCTION}

Cancer is one of the leading causes of death worldwide [Hyuna Sung et al. (2020), Freddie Bray et al. (2021)]. The Global Cancer Observatory (GLOBOCAN) 2020 predicts that in the year 2040, there will be 28.4 million new cases of cancer. Compared to the 19.3 million new cases reported in 2020 [GLOBOCAN (2020)], a 47 percent increase. Surgery, chemotherapy, radiotherapy, immunotherapy, hormone therapy, and gene therapy are just a few treatment options available for cancer. When used alone or in conjunction with chemotherapy and surgery, radiotherapy is critical in many cancer cases, whether the goal is curative or palliative. About half of cancer patients receive radiotherapy [Byron Burnette et al. (2013), Basker. R et al. (2017)]. Radiation entered the medical field as soon as Wilhelm Conard Rontgen in 1895 and Henri Becquerel in 1896 discovered X-rays and radioactivity. Much energy needs to be put into processes that damage DNA (Deoxyribonucleic Acid) in the body, such as ionization and excitation (such as X-rays, gamma rays, and carbon ions). Trial-and-error research produced brachytherapy and external beam radiotherapy, both developed due to collaboration between scientists and clinicians.

EBRT (external beam radiotherapy) and brachytherapy are the two main types of radiotherapy used in cancer treatment. In external beam radiotherapy, either a high-energy X-ray beam or light ion beam directs the patient from outside the body. In brachytherapy, the radioactive source is placed inside the tumor or near the tumor. There are only a few instances in which brachytherapy is utilized instead of external beam radiotherapy (EBRT). The external beam radiotherapy was started with kilovoltage therapy in the 1920s [Van Dyk. J et al. (2020)]. There was a limitation of less penetration and skin-sparing. Securing the radioactive source via sealed radioactive teletherapy is also an option for treating the problem. The Betatron (Megavoltage X-rays and electrons) and Microtron (Megavoltage X-rays and electrons) also were used in external beam radiotherapy.
External beam radiotherapy reached a milestone with the invention of Cobalt teletherapy in 1950 as it was able to provide increased photon penetration and skin-sparing. The first patient was treated with a Cobalt-60 machine in 1951 in Ontario, Canada [Van Dyk. J et al. (2020)]. The energy of Cobalt teletherapy is typically $1.25 \mathrm{MV}$, and the maximum dose $\left(D_{\max }\right)$ will be at $0.5 \mathrm{~cm}$. Cobalt teletherapy treatment was better due to gamma rays' low megavoltage energy. It is ideal for treating head and neck cancers, breast cancers, and some types of superficial Sarcomas. But, for other cancer cases like the cervix, esophagus, lung, prostate, etc., the treating area's thickness would usually be greater than $20 \mathrm{~cm}$. It will require complicated treatment plans to achieve the desirable isodose distribution.

During the remarkable growth of Cobalt teletherapy, the medical linear accelerator was introduced in 1952 [David. I and John. B (2006)]. There was a strong competition between Cobalt teletherapy and Linac. Linac is equipment that uses high frequency electromagnetic (EM) waves to accelerate electrons/charged particles to high energies. It can work in a dual-mode using high-energy electrons to treat superficial tumors. These high-energy electrons hit a target to produce high-energy photon beams. Medical Linacs replaced Cobalt teletherapy machines in many developed countries in the 1970s and 1980s due to rapid progress in Linac technology, such as multi-leaf collimators (MLCs), intensity modulation, and image guidance [Van Dyk. J et al. (2020)]. One major drawback in using radiotherapy is that healthy tissue can also be affected during the radiation treatment. Therefore, the foremost goal of radiation therapy is to precisely target the tumor volume with minimal exposure to the surrounding normal healthy tissue [Ramanathan V (2017)]. Newly introduced radiotherapy technology regularly helped patients to reach their treatment goals. Radiation therapy is advancing rapidly in terms of technology. Presently, particle therapy (proton, carbon ion, etc.) has been increasingly popular due to the steep dose fall-off (Bragg peak) depth dose profile [Ramanathan $\mathrm{V}$ and Peterson S (2019)]. 
Modern radiotherapy devices require very complex treatment techniques and exact applications. Therefore, a highly qualified multidisciplinary medical professional in the field of radiation oncology is required. Moreover, purchasing modern radiotherapy equipment, maintenance of radiotherapy equipment, and training health professionals are costly processes. Therefore, using modern radiotherapy equipment in cancer treatment is a challenging task for developing countries. Meantime, the new cancer cases are dramatically increasing. In this scenario, the Cobalt-60 teletherapy machine helps many low- and middle-income countries in cancer care.

The current view about Cobalt teletherapy is that it is an old treatment modality. It can be only applicable for palliative care or valuable for low- and middleincome countries with limited resources. Cobalt-60 teletherapy with multi-leaf collimators also was introduced to provide intensity-modulated radiotherapy. It was successful, but it was unable to compete with medical linac's versatile technology. The significant problems with Cobalt teletherapy are lack of sharpness of the beam edge due to the size of the Cobalt source and radiation penumbra, which hinder the confinement of the dose to tumor volume, and decaying radioactive Cobalt source, which leads to reduced output. The treatment time needs extended time as a result of the decreased output. In addition, the source should be replaced every 5 to 7 years because the half-life of Cobalt- 60 is 5.3 years, and the disposal of the decayed source is another major problem.

Moreover, the transport cost for Cobalt-60 radioactive sources and other radioactive sources used for medical and industrial purposes is expensive, and the transport process is also very complicated. Because of its simplicity, relatively low initial cost, lack of Quality Assurance (QA), and lower service charge than linear accelerators, Cobalt teletherapy machines still contribute to cancer care even though they have limitations. It is especially true in low- and middle-income countries. This study sought to determine how Cobalt teletherapy machines contribute to cancer care in the modernized radiotherapy era.

\section{METHODS}

Since its foundation, the International Atomic Agency (IAEA) has maintained an inventory of radiotherapy centers and radiotherapy equipment to help its member states. The DIRAC (Directory of Radiotherapy Centres) has established a database of more than 60 years of continuous work collaborating between IAEA and health professionals worldwide. At present, the DIRAC database comprises about $90 \%$ of globally available radiotherapy facilities. Mostly, all the updated data are according to the present status of facilities in each country [DIRAC (2021)]. In addition, the IAEA-DIRAC database offers the geographical distribution of radiotherapy facilities worldwide. The DIRAC database helps identify the gaps in the availability of radiotherapy equipment and calculate the needs of radiotherapy worldwide.

Globally available external beam radiotherapy facilities were identified using the IAEA-DIRAC database. North America, Mexico, Central America, Tropical South America, Temperate South America (including the Caribbean), Eastern Europe (including the Baltic States), and Northern Asia (including the Caucasus) consist of the most countries with radiotherapy facilities. Other regions with radiotherapy facilities include North Africa (including sub-Saharan Africa) and the Middle East (including the Arabian Peninsula). We counted the number of linear accelerators and light-ion therapy machines used in each DIRAC country. We also looked at the number of Cobalt teletherapy machines in use. According to the observations, all countries of the DIRAC region had varying income levels.

The information categorized into four groups helped analyze the differences between the various income levels: high-income countries, upper and lowermiddle-income countries, and low and middle incomes. The mean megavoltage units per million people for each DIRAC region was calculated based on per capita income using the total number of external beam teletherapy machines (cobalt teletherapy and linear accelerator) and the 


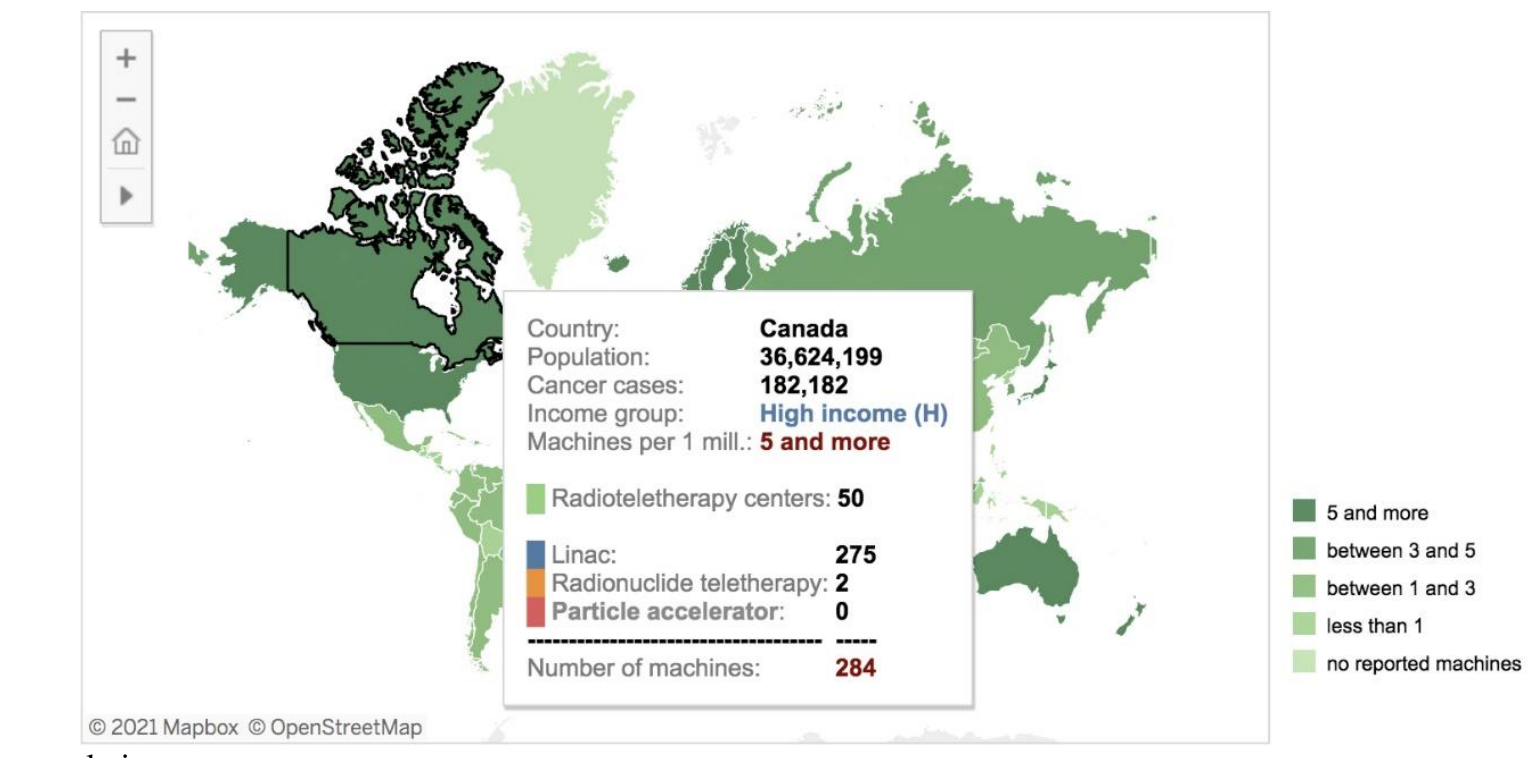

population.

Figure 1: Access to radiotherapy in Canada

It is vital to determine the number of Cobalt teletherapy machines per million people. The calculation proceeded by using the population and the available, total number of Cobalt teletherapy machines. Figure 1 shows the collection of data in Canada. The technique is similar for all other countries.

The observations indicated the distribution of linear accelerators and Cobalt teletherapy in high-income countries and low- and middle-income countries in 2006, 2013, and 2020 [DIRAC (2021), IAEA (2017)]. OriginPro data analysis program [OriginLab (2021)] helped the analysis of the future scope of Cobalt teletherapy in developed and developing countries.

\section{RESULTS AND DISCUSSION}

According to the DIRAC database, as of April 2021, only 145 countries out of 214 had radiotherapy facilities. There is no access to radiotherapy facilities in 51 countries, including 30 in Africa, that have no radiotherapy and depend on either non-radiotherapy treatment options or neighboring countries [IAEA (2017)].

Table 1 shows a summary of details on the availability of radiotherapy facilities in 145 countries.
There is a total of 7610 radiotherapy centers worldwide. There are currently 13932 teletherapy units in use, with linear accelerators accounting for $86.6 \%$. Cobalt teletherapy accounted for $12.7 \%$ of those, and light ion therapy accounted for the remaining $0.7 \%$. Because light ion therapy necessitates a substantial financial commitment, it was limited to just 20 countries. The number of light ion therapy facilities in the United States (37) and Japan (23) is higher. India is the only country in South Asia that offers light ion therapy (also known as proton therapy).

Table 1: Globally available EBRT facilities

\begin{tabular}{|l|l|}
\hline Detail & Total number \\
\hline Radiotherapy treatment centers & 7610 \\
\hline Teletherapy megavoltage units & 13932 \\
\hline Linear accelerators & 12063 \\
\hline Cobalt teletherapy machines & 1766 \\
\hline Light ion therapy & 103 \\
\hline
\end{tabular}

Table 2 details the availability of external beam radiotherapy equipment (Cobalt teletherapy and linear accelerators) organized by the DIRAC region and income group. When analyzing the availability of radiotherapy facilities by a million population, we can understand the distribution of radiotherapy machines worldwide. 
Table 2 [part A]: Availability of megavoltage teletherapy facilities. Notations indicate as follows: HIC - High-Income Countries, UMIC - Upper Middle-Income Countries, LMIC- Lower Middle-Income Countries, LIC - Lower-Income Countries, RT - Radiotherapy, and MV - Mega Voltage.

\begin{tabular}{|c|c|c|c|c|}
\hline $\begin{array}{l}\text { DIRAC region } \\
\text { and Income } \\
\text { group }\end{array}$ & $\begin{array}{l}\text { Cobalt } \\
\text { teletherapy }\end{array}$ & $\begin{array}{l}\text { Linear } \\
\text { accelerators }\end{array}$ & $\begin{array}{l}\text { MV machines per million } \\
\text { population (mean) }\end{array}$ & $\begin{array}{l}\text { Contribution of Cobalt teletherapy to } \\
\text { MV per million population } \\
\text { (mean) }\end{array}$ \\
\hline \multicolumn{5}{|c|}{ NORTH AMERICA } \\
\hline \multicolumn{5}{|c|}{ Countries (2) and Countries with RT (2) } \\
\hline $\mathrm{HIC} \mathrm{(2)}$ & 21 & 3715 & 9.11 & 0.05 \\
\hline \multicolumn{5}{|c|}{ MEXICO AND CENTRAL AMERICA } \\
\hline \multicolumn{5}{|c|}{ Countries (8) and Countries with RT (7) } \\
\hline HIC (1) & 0 & 8 & 1.95 & 0 \\
\hline UMIC (4) & 24 & 153 & 0.89 & 0.12 \\
\hline LMIC (3) & 9 & 10 & 0.88 & 0.42 \\
\hline \multicolumn{5}{|c|}{ TROPICAL SOUTH AMERICA } \\
\hline \multicolumn{5}{|c|}{ Countries (10) and Countries with RT (9) } \\
\hline UMIC (8) & 94 & 501 & 1.78 & 0.28 \\
\hline LMIC (1) & 5 & 3 & 0.72 & 0.45 \\
\hline \multicolumn{5}{|c|}{ TEMPERATE SOUTH AMERICA } \\
\hline \multicolumn{5}{|c|}{ Countries (3) and Countries with RT (3) } \\
\hline $\mathrm{HIC} \mathrm{(2)}$ & 2 & 52 & 2.78 & 0.10 \\
\hline UMIC (1) & 31 & 98 & 2.91 & 0.70 \\
\hline \multicolumn{5}{|c|}{ CARIBBEAN } \\
\hline \multicolumn{5}{|c|}{ Countries (20) and Countries with RT (10) } \\
\hline HIC (7) & 3 & 32 & 5.39 & 0.46 \\
\hline UMIC (3) & 14 & 28 & 1.69 & 0.56 \\
\hline \multicolumn{5}{|c|}{ WESTERN EUROPE } \\
\hline \multicolumn{5}{|c|}{ Countries (26) and Countries with RT (19) } \\
\hline HIC (19) & 27 & 2750 & 7.04 & 0.07 \\
\hline \multicolumn{5}{|c|}{ EASTERN EUROPE AND NORTHERN ASIA } \\
\hline \multicolumn{5}{|c|}{ Countries (29) and Countries with RT (28) } \\
\hline HIC (10) & 41 & 255 & 3.71 & 0.51 \\
\hline UMIC (12) & 337 & 353 & 2.89 & 1.41 \\
\hline LMIC (5) & 9 & 151 & 1.22 & 0.07 \\
\hline LIC (1) & 6 & 1 & 0.22 & 0.19 \\
\hline \multicolumn{5}{|c|}{ NORTH AFRICA } \\
\hline \multicolumn{5}{|c|}{ Countries (6) and Countries with RT (5) } \\
\hline UMIC (1) & 11 & 13 & 2.08 & 0.95 \\
\hline LMIC (4) & 26 & 176 & 1.06 & 0.14 \\
\hline
\end{tabular}


Table 2 [part B]: Availability of megavoltage teletherapy facilities. Notations indicate as follows: HIC - High-Income Countries, UMIC - Upper Middle-Income Countries, LMIC- Lower Middle-Income Countries, LIC - Lower-Income Countries, RT Radiotherapy, and MV - Mega Voltage.

\begin{tabular}{|c|c|c|c|c|}
\hline $\begin{array}{l}\text { DIRAC region } \\
\text { and Income } \\
\text { group }\end{array}$ & $\begin{array}{l}\text { Cobalt } \\
\text { teletherapy }\end{array}$ & $\begin{array}{l}\text { Linear } \\
\text { accelerators }\end{array}$ & $\begin{array}{l}\text { MV machines per million } \\
\text { population (mean) }\end{array}$ & $\begin{array}{l}\text { Contribution of Cobalt teletherapy to } \\
\text { MV per million population } \\
\text { (mean) }\end{array}$ \\
\hline \multicolumn{5}{|c|}{ MIDDLE AFRICA } \\
\hline \multicolumn{5}{|c|}{ Countries (45) and Countries with RT (21) } \\
\hline $\mathrm{HIC} \mathrm{(2)}$ & 2 & 3 & 2.33 & 0.93 \\
\hline LMIC (6) & 18 & 5 & 0.18 & 0.04 \\
\hline LIC (13) & 16 & 27 & 0.16 & 0.06 \\
\hline \multicolumn{5}{|c|}{ SOUTHERN AFRICA } \\
\hline \multicolumn{5}{|c|}{ Countries (6) and Countries with RT (4) } \\
\hline UMIC (2) & 1 & 8 & 0.61 & 0.07 \\
\hline LMIC (2) & 3 & 101 & 1.13 & 0.03 \\
\hline \multicolumn{5}{|c|}{ MIDDLE EAST } \\
\hline \multicolumn{5}{|c|}{ Countries (15) and Countries with RT (15) } \\
\hline $\mathrm{HIC} \mathrm{(8)}$ & 12 & 414 & 1.68 & 0.05 \\
\hline UMIC (4) & 8 & 88 & 1.54 & 0.13 \\
\hline LIC (3) & 1 & 7 & 2.13 & 0.21 \\
\hline \multicolumn{5}{|c|}{ SOUTH ASIA } \\
\hline \multicolumn{5}{|c|}{ Countries (8) and Countries with RT (5) } \\
\hline LMIC (5) & 396 & 377 & 0.44 & 0.23 \\
\hline \multicolumn{5}{|c|}{ EAST ASIA } \\
\hline \multicolumn{5}{|c|}{ Countries (8) and Countries with RT (5) } \\
\hline HIC (4) & 580 & 2210 & 3.01 & 0.63 \\
\hline UMIC (1) & 3 & 2 & 1.63 & 0.98 \\
\hline \multicolumn{5}{|c|}{ SOUTHEAST ASIA } \\
\hline \multicolumn{5}{|c|}{ Countries (15) and Countries with RT (9) } \\
\hline HIC (1) & 0 & 2 & 4.67 & 0 \\
\hline UMIC (3) & 6 & 77 & 0.80 & 0.06 \\
\hline LMIC (5) & 59 & 198 & 0.56 & 0.13 \\
\hline \multicolumn{5}{|c|}{ SOUTHERN AND WESTERN PACIFIC } \\
\hline \multicolumn{5}{|c|}{ Countries (13) and Countries with RT (3) } \\
\hline HIC (2) & 0 & 245 & 7.42 & 0 \\
\hline LMIC (1) & 1 & 0 & 0.12 & 0.12 \\
\hline
\end{tabular}


If the megavoltage machine per million population's access is very low, the patients have to wait long to receive radiotherapy treatment. It simply means that there is no access to radiotherapy. An internationally recognized radiotherapy facility is one megavoltage machine per 250,000 people [Surbhi Grover et al. (2015)].

Megavoltage machines per million people are most prevalent in North America (9.11), while low-income countries in the South and Western Pacific have the fewest (0.12). The analysis is due to the global distribution of megavoltage machines. The Cobalt teletherapy contribution is in the range of 0 to 1.41 megavoltage machines per population. East and northern Europe and Asia's upper-middle-income countries use Cobalt teletherapy the most (1.41 megavolt machines per million people). There is no Cobalt teletherapy usage in high-income countries of Mexico and Central America, Southeast Asia, and the Southern and Western Pacific. Generally, the usage of Cobalt teletherapy in high-income countries is less than that of low- and middle-income countries.

There are considerable disparities in radiotherapy access worldwide. Overall, there are significant shortfalls in radiotherapy facilities except in some high-income countries. Due to a dramatic lack of economic and human resources, most low- and middle-income countries are either unable to develop new radiotherapy facilities or expand their radiotherapy facilities to IAEA recommended levels of radiotherapy facilities. For example, in Nigeria, only one Cobalt teletherapy unit is available for about 100 million people. Using that Cobalt teletherapy machine for more than five years without replacing the Cobalt source will lead to a longer treatment time. Longer treatment time is uncomfortable for the patients. Also, increased treatment time leads to inaccuracy in treatment as the patients cannot be laid down on the treatment couch for a long time.

Figure 2 shows the distribution of Cobalt teletherapy units per income group of the world. The wealthy countries own more than two-thirds of the world's cobalt teletherapy devices, with the poorer countries owning the remaining one-third. The world's cobalt teletherapy machines are in countries with low income in $0.86 \%$ of cases.

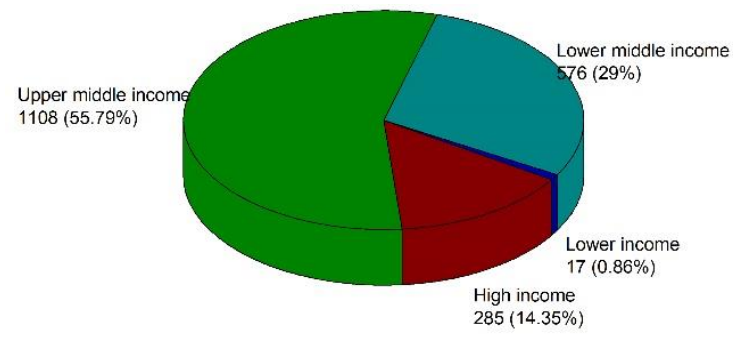

Figure 2: Distribution of Cobalt teletherapy units per income group of the world.

Figure 3 shows the disparities in the profile of existing linear accelerators and Cobalt teletherapy machines in developed and developing countries in the last two decades. In 2006, usage of Linacs was $85.3 \%$ in developed countries while only $37.3 \%$ Linac usage in developing countries. In 2013, Linacs usage in developed countries was $95.5 \%$, whereas that of developing countries was 58\%. In 2020, Linacs usage in developed countries was $96.8 \%$, and in developing countries, it was $69.4 \%$.

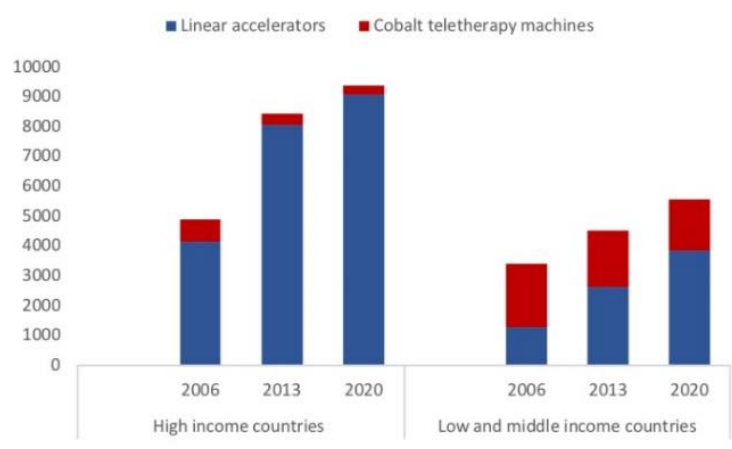

Figure 3: Distribution of linear accelerators and Cobalt teletherapy machines considering the level of income group.

Looking at the trend of Linacs in the last two decades, usage of Linacs gradually increased while usage of Cobalt teletherapy decreased both in developed and developing countries. Because of Linac's versatile technology, which provides conformal radiotherapy, increased accuracy, and low toxicity, most radiotherapy centres have switched from Cobalt teletherapy units [Van Dyk. J et al. (2020)]. At present, the installation and maintenance for lowenergy Linacs are compatible with Cobalt teletherapy. Therefore, making installation of new Cobalt teletherapy is less likely in the future. But, some large radiotherapy centres in low- and middle- 
income countries will invariably retain the Cobalt teletherapy machines for at least the next decade for palliative radiotherapy in the management of increasing cancer care.

\section{CONCLUSION}

The role of Cobalt teletherapy is considerable in lowand middle-income countries. Currently, available Cobalt teletherapy units are 1766 , which is $12.7 \%$ of available teletherapy units globally. 86 percent of Cobalt teletherapy units are located in developing countries. There are significant disparities in megavoltage machines per million populations worldwide. From 0.12 to 9.11 megavolt machines per million people are available, with a Cobalt teletherapy contribution between 0 and 1.41. In teletherapy, developed countries currently have 96.7 percent of the Linacs used; however, developing countries currently have 69.5 percent of the Linacs used, down from $37.3 \%$ in 2006 . Overall, the trend of using Cobalt teletherapy for curative treatment is gradually decreasing even in developing countries. For palliative treatment, large radiotherapy treatment centres may continue to use Cobalt teletherapy. A considerable number of radiotherapy treatment centres in low- and middle-income countries will have to use Cobalt teletherapy machines to manage dramatically increasing new cancer cases if they could expand their facilities in radiotherapy. The use of Cobalt teletherapy in the future is unavoidable in low- and middle-income countries.

\section{REFERENCES}

Hyuna Sung; Jacques Ferlay; Rebeecca L Siegel; Mathieu Laversanne; Isabelle Soeriomataram; Ahmedin Jemal and Freddie Bray (2021). Global cancer statistics 2020: GLOBOCAN estimates of incidence and mortality worldwide for 36 cancers in 185 countries, CA: A Cancer Journal for Clinicians 71(3) pp 209-249.

Freddie Bray; Mathieu Laversanne; Elisabete Weiderpass and Isabelle Soeriomataram (2021). The ever-increasing importance of cancer as a leading cause of premature death worldwide, Cancer 127(16) pp 3029-3030.

\section{Cancer Today-Global Cancer Observatory (GLOBOCAN) - $\quad$ IARC, https://gco.iarc.fr/today/home [Accessed 20 ${ }^{\text {th }}$ June 2021].}

Byron Burnette; Ralph, R Weichselbaum (2013). Radiation as an immune modulator, Seminars in Radiation Oncology, 23(4), pp 273-280.

Basker, R; Itahana, K (2017). Radiation therapy and cancer control in developing countries: Can we save more lives? Int. J. Med. Sci. 14(1), pp 13-17

Van Dyk, J; Battista, J J, and Almond, P R (2020). A retrospective of Cobalt-60 radiation therapy: "The atom bomb that saves lives"' Medical Physics International Journal, Special Issue, History of Medical Physics 4.

David I Thwaites and John B Tuohy (2006). Back to the future: the history and development of the clinical linear accelerator, Phys. Med.Biol, 51 R343.

Ramanathan, V (2017). Measuring cross-section data for prompt gammas emitted during proton nucleus collisions, (South Africa: University of Cape Town) Ph.D. Thesis.

Ramanathan, V, and Peterson, S (2019). Investigating the aberration found in $4.438 \mathrm{MeV}{ }^{12} \mathrm{C}$ photo peak of the Geant 4 simulated prompt gamma spectrum, J.Phys.:Conf.Ser. 1248012041.

International Atomic Energy Agency, DIRACDirectory of Radiotherapy Centres, https://dirac.iaea.org [accessed $20^{\text {th }}$ May 2021]

Radiotherapy in cancer care: Facing the global challenge, International Atomic Energy Agency Publication, 2017.

Origin: Data Analysis and Graphing Software OriginLab, https://www.originlab.com/orogin [accessed 30th May 2021].

Surbhi Grover, Melody J, Xu, Alyssa Yeager, Lori Rosman, Reinou S Groen, Smita Chackungal, Danielle Rodi, Margaret Mangaali, Sommer Nurkic, 
Annemarie Fernandes, Lilie L Lin, Gillian Thomas, Anal Tergas (2015).11.2021 A systematic review of radiotherapy capacity in low-and middle- income countries, Frontiers in Oncology; https://doi;10.3389/fonc.2014.00380. 